\title{
A Low Noise, Wide Dynamic Range TOF Laser Radar Receiver Based on Pulse Shaping Techniques
}

\author{
Aram Baharmast, Tarmo Ruotsalainen and Juha Kostamovaara \\ University of Oulu, ITEE Faculty \\ Circuits and Systems Research Unit (CAS) \\ Oulu, Finland \\ aram.baharmast@oulu.fi, tarmo.ruotsalainen@oulu.fi,juha.kostamovaara@oulu.fi
}

\begin{abstract}
A time of flight (TOF) laser radar receiver based on unipolar-to-bipolar pulse shaping at its input is presented. The pulse shaping and the non-linear feedback of the transimpedance preamplifier give low timing error and jitter over a wide input pulse amplitude range. This receiver is realized in a $0.35 \mu \mathrm{m}$ CMOS technology and intended to be used in laser ranging with laser pulses of width $\sim 1$ ns. Post-layout simulations show a dynamic range of more than 1:200000, a trans-impedance gain of $117 \mathrm{db} \Omega$, a bandwidth of $260 \mathrm{MHz}$ and an input-referred equivalent current noise of 70nA. These results are achieved while keeping the walk error less than $\pm 50 \mathrm{ps}(7.5 \mathrm{~mm})$ without any need for complicated calibration methods.
\end{abstract}

Keywords-time-of-flight (TOF); trans-impedance amplifier (TIA); pulse shaping technique; walk error; dynamic range (DR); laser radar receiver

\section{INTRODUCTION}

The pulsed TOF laser distance measurement technique is based on measurement of the travel time of a light pulse from the transmitter to the observed object and back to the receiver [1]. The benefits of pulsed TOF laser radar techniques over microwave and ultrasonic radars lie in the ease of controlling the optical measurement beam with lenses and the stability of the speed of light. Furthermore, unambiguous single-shot measurement results with at $\mathrm{cm}$ level or even millimetre-level precision are attainable at a high rate (e.g. $10-100 \mathrm{kHz}$ ) which is not possible with continuous-wave measurement techniques, for example [2].

A typical TOF system consists of a laser pulse transmitter (to send high power laser pulses), optics (to illuminate the object and to collect the reflected echoes), a photo-detector (usually an avalanche photodiode (APD)), a receiver channel (to detect the weak optical echo pulse and extract the timing moment from the received pulse) and a time interval measurement unit (TDC). The timing jitter generated by the noise and the systematic timing walk error are two important sources of inaccuracy in TOF systems. Jitter, i.e. uncertainty in the timing point, randomly affects the timing detection on the receiver side, as shown in Fig. 1. This effect is proportional to the rise time of the arriving pulse and inversely proportional to the signal-to-noise ratio at the input of the receiver [3, 4]. The timing walk error is the systematic deviation of the timing point with pulse variations, its main sources being variation in the pulse rise time (geometric walk error, Fig. 1b) and variations in the electronic delay through the receiver channel at different input amplitudes (Fig.1c) $[5,6]$.
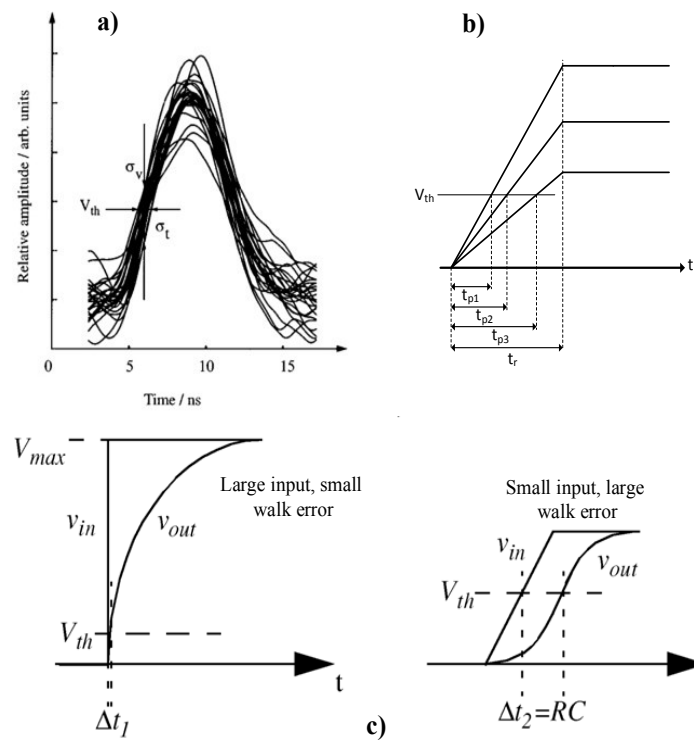

Fig. 1 Various sources of inaccuracy in TOF receivers, a) the effect of timing jitter, b) geometric walk error, c) walk error due to change in the electronic delay in the receiver channel

The capability for working over a wide DR is another important parameter (related to the timing walk error) in the design of TOF receivers, since the DR of the optical input signal (the ratio of the maximum to the minimum signal current peak value at the output from the optical detector) can be as wide as 1:10 000 or even more, depending on the distance, reflectivity and angle of the object and on weather conditions.

Various techniques have been proposed for implementing a TOF receiver, and the state-of-the-art solutions include compensated leading edge detection ([7-9]), linear range detection (using gain control, $[3,10]$ ) and unipolar-to-bipolar conversion ([11, 12]). Even though the first method is capable of achieving a high DR, it is relatively complex due to the complicated software-based calibration methods employed in it. The second technique suffers from a limited DR (1 to 500-1500), which arises from the non-constant electric delay in the gain control electronics.

In the third technique, the first zero crossing point of the converted signal is picked out as the timing moment. As a result, if the receiver channel is fast enough to recover from saturation, the clipping of the signal that occurs at high amplitudes does not affect the timing moment. Even though 
this approach is capable of operating over a wide DR and with a low walk error in principle, the implementations proposed so far, undergo various limitations. In [12] the received signal from the APD is amplified using first a regulated cascaded current buffer (RGC) and then a high pass $\mathrm{RC}$ differentiator is adopted to convert the unipolar pulse to a bipolar signal. In this case the limited DR of the RGC limits that of the whole receiver channel. Furthermore, this conversion technique cannot be implemented next to the APD due to the large capacitance of the photodiode and I/O pad which corrupts the conversion.

A different implementation of the unipolar-to-bipolar conversion receiver was proposed in [11], using an RLC resonator at the input of the receiver. Even though this technique imposes no specific conditions on the received signal at the high end, the DR is restricted at low end due to the excessive noise generated by the bias and damping resistors.

This paper presents a new implementation of a TOF receiver channel based on the unipolar-to-bipolar conversion technique. In this method an LC pulse shaper and modified shunt feedback TIA are combined to realize a low noise, wide DR receiver, without using any complicated calibration methods. This receiver has been designed to detect laser pulses with a width and rise time of $\sim 1$ ns using an avalanche photodiode (APD) as the photodetector. The paper is organized as follows: The operation principle is discussed in section II, the receiver design details are described in section III, simulation results are shown in section IV and conclusions are given in Section V.

\section{OPERATION PRINCIPLE}

The basic block diagram of the proposed TIA-based pulse shaping technique is shown in Fig. 2. In this technique, the inductor (L) is adopted both to bias of the APD and as a part of the RLC resonator network, and no bias resistors are used. The feedback resistor of the TIA $\left(R_{F}\right)$ acts both as the damping element of the RLC resonator and as a pass to convert the current generated by the pulse shaping unit to a voltage at the output of the TIA. Therefore due to the absence of front end resistors and the low noise gain (because of the increasing impedance of the inductor), low noise behaviour can be expected at high frequencies. The operation principle and design details of this front end are described in [13].

The parallel RLC resonator should provide an underdamping response. The required damping is provided by the resistance seen at the input of the TIA $\left(R_{\text {in,TIA }}=\right.$ $R_{F} /\left(A_{0}+1\right)$ ), where $A_{0}$ is the gain of the core amplifier (A)). Moreover, the values of $L, R_{F}$ and $A_{0}$ are chosen according to the required bandwidth and minimization of the walk error and noise.

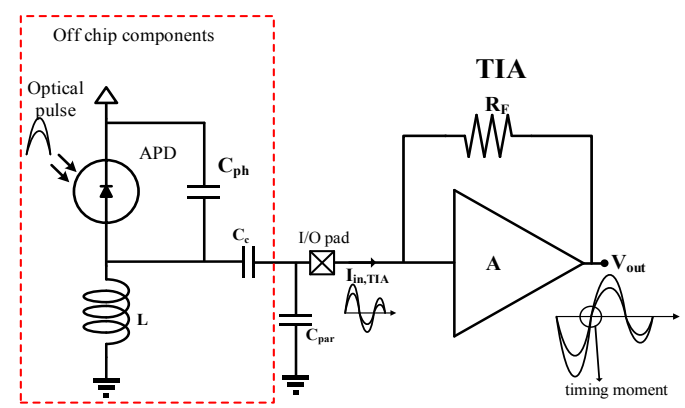

Fig. 2 A simple model for the proposed unipolar to bipolar conversion technique

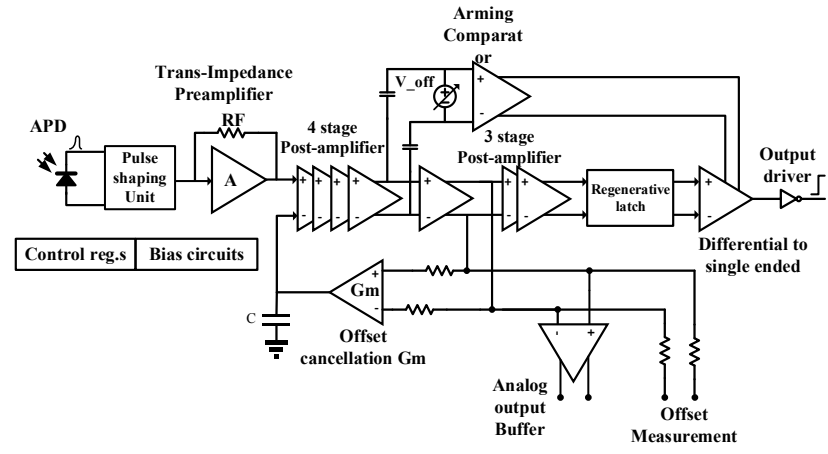

Fig. 3 The block diagram of the whole receiver

In the leading edge TOF receivers the rise time of the arriving pulse determines the bandwidth required, as in ([3]):

$$
B W=\frac{0.35}{t_{\text {rise }}}
$$

However, simulations show that a lower bandwidth is sufficient for our proposed receiver. Since the target in this project is to detect pulses with a width and rise time of around $1 \mathrm{~ns}$ or more, a bandwidth of less than $300 \mathrm{MHz}$ is required. The bandwidth of the whole receiver channel is specified by the dominant pole of the TIA, which is located at its input node $[13,14]$ :

$$
\omega_{-3 \mathrm{~dB}} \cong \frac{A_{0}+1}{2 R_{\mathrm{F}} C_{T}}+\sqrt{\frac{1}{L C_{T}}+\left(\frac{A_{0}+1}{2 R_{\mathrm{F}} C_{T}}\right)^{2}}
$$

where $C_{T}$ is the total input node capacitance. The value of $C_{T}$, including the photodiode $(\sim 0.5 p F)$ and the parasitic capacitance of the input pad and bonding wires $(\sim 3 p F)$, critically affects the performance of the system regarding the noise level and the walk error.

\section{RECEIVER CHANNEL DESIGN}

A block diagram of the whole receiver channel is depicted in Fig. 3. The detected echo pulse from the APD is converted to a bipolar voltage signal through the pulse shaping unit and TIA. The converted pulse is further amplified through seven wide-bandwidth differential amplifiers, and the amplified signal is applied to a crosscoupled regenerative latch to generate a digital-like pulse. This pulse is sent out from the chip through a differential-tosingle-ended structure and a chain of inverters for the time interval measurement with a time-to-digital converter (TDC) unit. An arming comparator is used to prevent the output from being excited by noise. The threshold level of the arming comparator is adjustable through a digital code from outside the chip. A feedback-based offset cancellation scheme is used to mitigate the offset through the differential amplifiers and ensure precise setting of the DC level at the input of the first post-amplifier.

\section{A. TIA}

The proposed TIA circuit realization is shown in Fig. 4. For better understanding, a block diagram of the core amplifier $(\mathrm{A})$ is also provided. The core amplifier is based on 


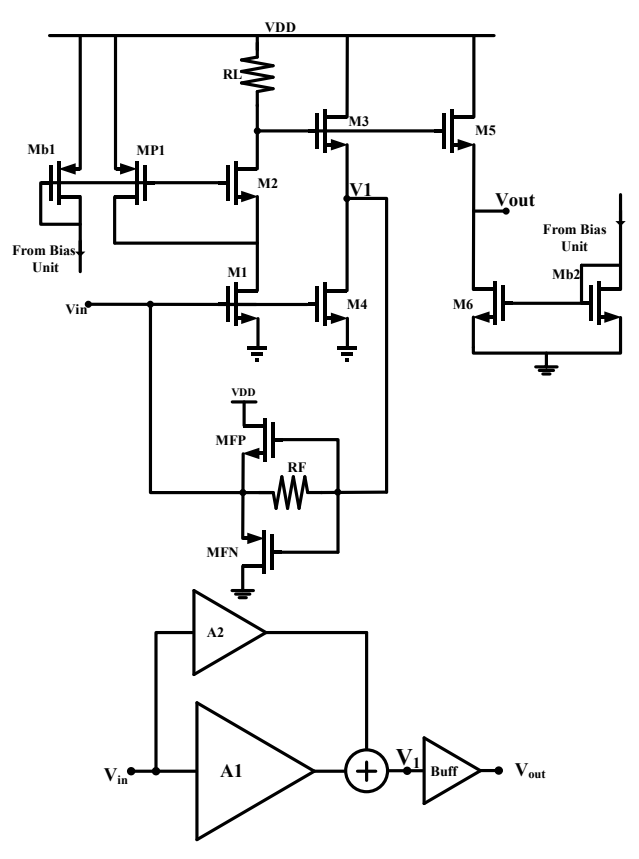

Fig. 4 Circuit level realization of the TIA and a simple block diagram of the core amplifier

feed-forward techniques implemented by amplifiers A1 and A2, which consist of a cascode stage (M1, M2 and RL) and a common source stage (M3, M4) respectively. In this realization $\mathrm{A} 1$ is the main gain stage, while $\mathrm{A} 2$ has a gain of around one and consequently saturates at larger input amplitudes and returns to the linear region sooner than A1. Simulations show that use of the simple cascode structure without feed-forwarding results in larger a walk error in the presence of a large $\mathrm{I} / \mathrm{O}$ pad and parasitic capacitance at the input node. This is due to the fact that for large input signals, when the TIA is saturated, A2 provides direct access to the output node $(V 1)$ from the input and speeds up recovery to the linear region.

Transistors MPF and MNF in the feedback pass play a key role in extending the DR of the receiver by preserving the damping behaviour of the TIA $\left(R_{F} /\left(A_{0}+1\right)\right)$ at large inputs. For small input currents $\left|V_{G S-M F N, M F P}\right| \leq\left|V_{T H}\right|$, these two transistors are switched off and have no effect on the signal shape. As the input current increases, they gradually turn on and sink the extra current from $V D D$ or source it to the ground [13]. Without these two transistors, the output signal of the TIA would tend to oscillate at large inputs due to lack sufficient damping.

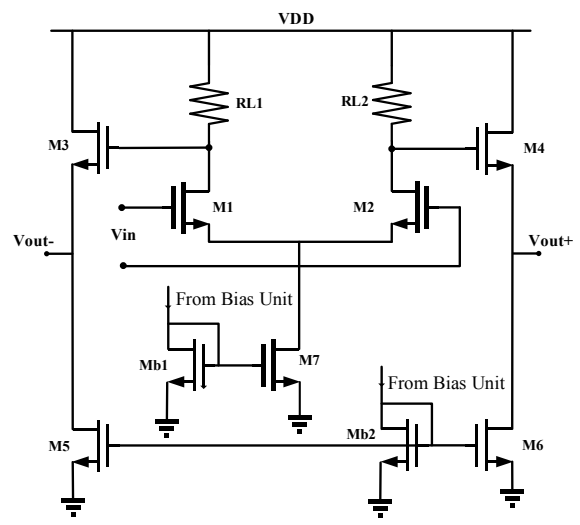

Fig. 5 Fully differential amplifier used in post amplifier stages
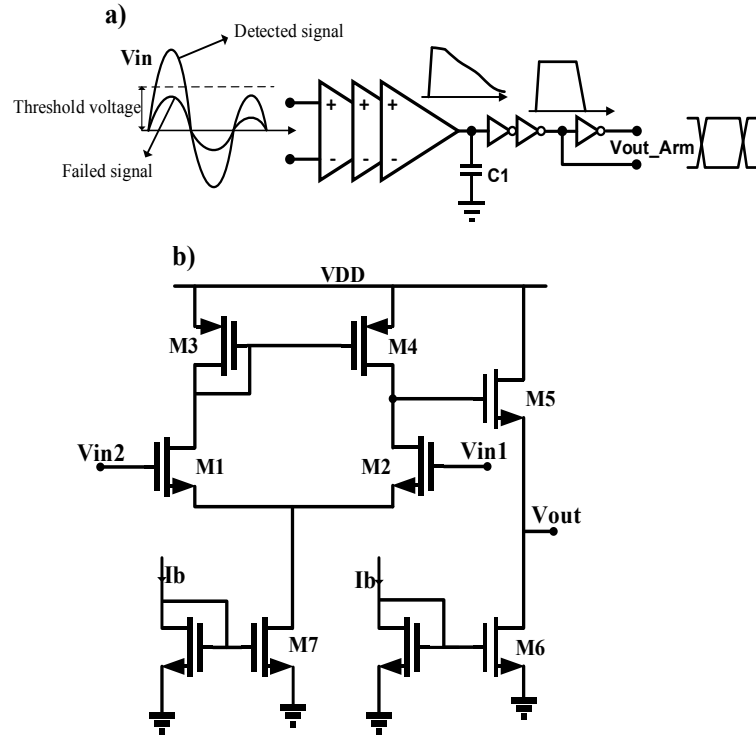

Fig. 6 a) The block diagram of the arming comparator and b) its third amplifier stage

The dominant noise sources of the whole receiver channel are the input transistor of the TIA (M1) and the feedback resistor $\left(R_{F}\right)$. Choosing the maximum $R_{F}$ based on the target bandwidth, the size of M1 should be large enough relative to the capacitance of the photodiode in order to minimize the noise peaking at high frequencies $([14,15])$.

\section{B. Post-amplifiers and analog output buffer}

Apart from the seventh post-amplifier, which uses a differential amplifier with a PMOS diode-connected load, the post-amplifiers are realized by simple wide bandwidth $(\sim 1 \mathrm{GHz})$, low gain $(\sim 10 \mathrm{~dB})$ differential amplifiers followed by source followers (Fig. 5). The bandwidth of the post-amplifiers is set to be high enough to recover from clipping well before the timing moment and low enough to minimize the noise through the channel. Use of the source followers simplifies the design of each stage and minimizes the loading effect from the following stage. The analog buffer that is added for measurement purposes is similar to the post-amplifiers (Fig. 5). Expect that resistors are used at the source of the input transistors to extend the linearity. The gain is around $2 \mathrm{~dB}$ and the bandwidth in the presence of load capacitance of the pad and external load model is $\sim 1 \mathrm{GHz}$.

\section{Arming comparator}

The structure of the arming comparator is shown in Fig. 6a. Its function is to enable output of the receiver only for those signals that exceed the present threshold. The first two stages of the arming comparator are fully differential amplifiers of the same structure as the post-amplifiers (Fig. 5), while the third amplifier that is shown in Fig. 6b, is a single-ended differential structure followed by a source follower stage. The bias current of the source follower $\left(I_{M 6}\right)$ is set to be an order of magnitude lower than the tail current of the input differential stage $\left(I_{M 7}\right)$ in order to charge the capacitor $(C 1)$ rapidly but discharge it slowly. The voltage of the capacitor is then fed to three inverter stages to produce a rail-to-rail sufficient wide voltage pulse. This technique 


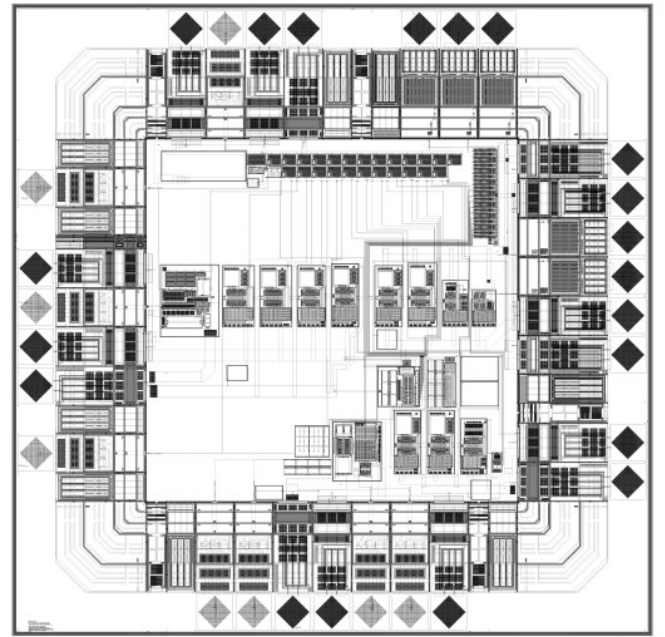

Fig. 7 The layout of the proposed receiver channel including I/O pads.

ensures proper synchronization of the timing of the arming signal with the output of the receiver at the final stage.

\section{Offset cancellation integrator}

The offset cancellation integrator tracks the average voltage difference at the output of the fourth amplifier and produces a compensating DC voltage to bias the input of the first post-amplifier, in order to minimize the offset through the amplifier channel. The high-speed signal is filtered using two large MOS resistors and a trans-conductance differential stage with a large capacitor (to produce a dominant pole for the feedback circuit). The large MOS resistors isolate the high-speed signal pass from the loading effect of the large input devices of the trans-conductance stage.

\section{SimULATION RESULTS}

The receiver channel was designed in a $0.35 \mu \mathrm{m}$ standard CMOS technology. The layout of the whole receiver channel submitted for fabrication is shown in Fig. 7. The occupied die area, including pads, is $1.7 \mathrm{~mm} \times 1.7 \mathrm{~mm}$. The whole receiver draws $62 \mathrm{~mA}$ current from a single $3.3 \mathrm{~V}$ supply voltage under normal conditions and consumes $73 \mathrm{~mA}$ when the analog output buffer is enabled. The total AC transimpedance gain up to the input of the arming comparator is $117 \mathrm{~dB} \Omega$, and the bandwidth (including the parasitic of the $\mathrm{I} / \mathrm{O}$ pads and bonding wires) is about $260 \mathrm{MHz}$. The total

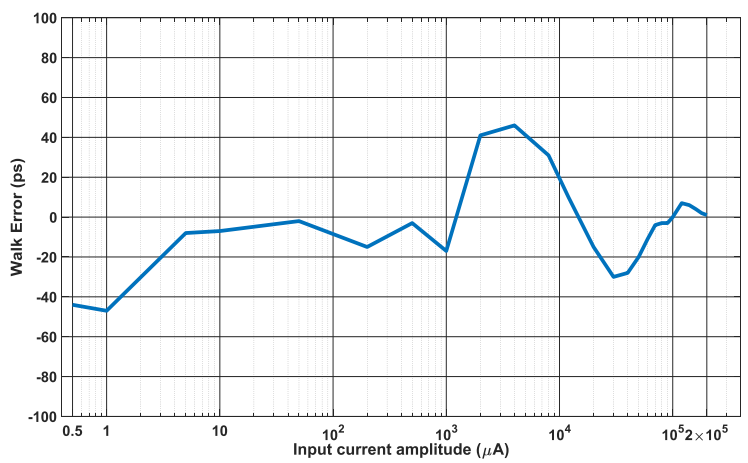

Fig. 8 A post-layout simulation of the walk error for the dynamic range 1:400000 simulated noise at the input of the arming comparator is $50 \mathrm{mV}$, or 70nA RMS when referred to the input. The walk error for the DR of the 1:400 $000(0.5 \mu \mathrm{A}: 200 \mathrm{~mA})$, as shown in Fig. 8, is less than $\pm 50 \mathrm{ps}$, or $7.5 \mathrm{~mm}$ in distance. The simulation results is summarized in Table 1.

The key point is that these results are achieved without any hardware or software compensation techniques. The state-of-the-art receiver of [8], for example, is capable of achieving a wide DR and a low walk error but requires a lookup table for the relation between walk error and pulse width/slew rate to compensate for the walk error in collaboration with the TDC unit. Another recent design, presented in [16], adopts a C-TIA to increase the sensitivity of the receiver in order to detect very weak optical pulses. It also uses constant-delay detection method for timing walk error compensation, however its DR is 1:12000 and its walk error is of the order of nanosecond.

Table 1 Summary of the post-layout simulation results

\begin{tabular}{c|c}
\hline Specification & Value \\
\hline Process & $0.35 \mu \mathrm{m}$ standard CMOS \\
\hline Supply voltage $(\mathrm{V})$ & 3.3 \\
\hline Current consumption $(\mathrm{mA})$ & 62 (Analog Buffer Off) \\
\cline { 2 - 2 } & 73 (Analog Buffer On) \\
\hline Bandwidth (MHz) & 260 \\
\hline Trans-impedance gain $(\mathrm{dB} \Omega)$ & 117 \\
\hline Input referred noise current $(\mathrm{nA})$ & 70 \\
\hline Minimum SNR & 7 \\
\hline DR & \pm 50
\end{tabular}

\section{CONCLUSION}

The new TOF receiver channel described here uses a combination of pulse shaping techniques and modified shunt feedback TIA to extend the DR range to more than 1:200000 and alleviate the noise level of the receiver to around $70 \mathrm{nA}$ while keeping the walk error less than $\pm 50 \mathrm{ps}$ without any specific calibration methods. The receiver has been designed and submitted for fabrication in a $0.35 \mu \mathrm{m}$ standard CMOS process.

\section{ACKNOWLEDGEMENTS}

The authors acknowledge financial support from the Academy of Finland (Centre of Excellence in Laser Scanning Research, contract no. 272196 and contracts nos. 255359, 263705 and 251571) and the Infotech Oulu Graduate School.

\section{REFERENCES}

S. Donati, Electro-optical instrumentation: sensing and measuring with lasers: Pearson Education, 2004.

P. Palojarvi, K. Maatta, and J. Kostamovaara, "Pulsed time-offlight laser radar module with millimeter-level accuracy using full custom receiver and TDC ASICs," IEEE Transactions on Instrumentation and Measurement, vol. 51, pp. 1102-1108, 2002.

[3] T. Ruotsalainen, P. Palojarvi, and J. Kostamovaara, "A wide dynamic range receiver channel for a pulsed time-of-flight laser radar," IEEE Journal of Solid-State Circuits, vol. 36, pp. 12281238,2001

[4] M. Simpson, C. Britton, A. Wintenberg, and G. Young, "An integrated, CMOS, constant-fraction timing discriminator for 
multichannel detector systems," IEEE transactions on nuclear science, vol. 42, pp. 762-766, 1995.

[5] P. Palojarvi, T. Ruotsalainen, and J. Kostamovaara, "A 250$\mathrm{MHz}$ BiCMOS receiver channel with leading edge timing discriminator for a pulsed time-of-flight laser rangefinder," IEEE Journal of solid-state circuits, vol. 40, pp. 1341-1349, 2005.

[6] R. J. Van De Plassche and P. Baltus, "An 8-bit 100-MHz fullNyquist analog-to-digital converter," IEEE Journal of SolidState Circuits, vol. 23, pp. 1334-1344, 1988.

[7] J. Nissinen, I. Nissinen, and J. Kostamovaara, "Integrated receiver including both receiver channel and TDC for a pulsed time-of-flight laser rangefinder with cm-level accuracy," IEEE journal of solid-state circuits, vol. 44, pp. 1486-1497, 2009.

[8] S. Kurtti, J. Nissinen, and J. Kostamovaara, "A Wide Dynamic Range CMOS Laser Radar Receiver With a Time-Domain Walk Error Compensation Scheme," IEEE Transactions on Circuits and Systems I: Regular Papers, vol. 64, pp. 550-561, 2017.

[9] J. Xiao, M. Lopez, X. Hu, J. Xiao, and F. Yan, "A continuous wavelet transform-based modulus maxima approach for the walk error compensation of pulsed time-of-flight laser rangefinders," Optik-International Journal for Light and Electron Optics, vol. 127, pp. 1980-1987, 2016.

[10] T.-H. Ngo, C.-H. Kim, Y. J. Kwon, J. S. Ko, D.-B. Kim, and H.H. Park, "Wideband receiver for a three-dimensional ranging LADAR system," IEEE Transactions on Circuits and Systems I: Regular Papers, vol. 60, pp. 448-456, 2013.

[11] J. Pehkonen, P. Palojarvi, and J. Kostamovaara, "Receiver channel with resonance-based timing detection for a laser range finder," IEEE Transactions on Circuits and Systems I: Regular Papers, vol. 53, pp. 569-577, 2006.

[12] S. Kurtti and J. Kostamovaara, "Laser radar receiver channel with timing detector based on front end unipolar-to-bipolar pulse shaping," IEEE Journal of Solid-State Circuits, vol. 44, pp. 835-847, 2009

[13] A. Baharmast and J. Kostamovaara, "A low noise front end trans-impedance amplifier channel for a pulsed time-of-flight laser radar," in Ph. D. Research in Microelectronics and Electronics (PRIME), 2017 13th Conference on, 2017, pp. 285288.

[14] E. Säckinger, Broadband circuits for optical fiber communication: John Wiley \& Sons, 2005.

[15] A. Abidi, "On the choice of optimum FET size in wide-band transimpedance amplifiers," Journal of lightwave technology, vol. 6, pp. 64-66, 1988.

[16] H.-S. Cho, C.-H. Kim, and S.-G. Lee, "A high-sensitivity and low-walk error LADAR receiver for military application," IEEE Transactions on Circuits and Systems I: Regular Papers, vol. 61, pp. 3007-3015, 2014 